\title{
Atmospheric forcing by ALADIN/MFSTEP and MFSTEP oriented tunings
}

\author{
R. Brožková ${ }^{1}$, M. Derková ${ }^{2}$, M. Belluš ${ }^{2}$, and A. Farda ${ }^{1}$ \\ ${ }^{1}$ Czech Hydrometeorological Institute, Prague, Czech Republic \\ ${ }^{2}$ Slovak Hydrometeorological Institute, Bratislava, Slovakia \\ Received: 31 March 2006 - Published in Ocean Sci. Discuss.: 24 May 2006 \\ Revised: 7 August 2006 - Accepted: 26 August 2006 - Published: 1 September 2006
}

\begin{abstract}
ALADIN/MFSTEP is a configuration of the numerical weather prediction (NWP) model ALADIN run in a dedicated real-time mode for the purposes of the MFSTEP Project. A special attention was paid to the quality of atmospheric fluxes used for the forcing of fine-scale oceanographic models. This paper describes the novelties applied in ALADIN/MFSTEP initiated by the MFSTEP demands, leading also to improvements in general weather forecasting.
\end{abstract}

\section{Introduction}

ALADIN (Aire Limitée Adaptation dynamique Développement INternational) is a limited area model based on the global NWP (Numerical Weather Prediction) system of IFS/ARPEGE (Integrated Forecasting System/Action de Recherche Petite Echelle Grande Echelle). Its complete scientific description is not available in a single paper, but readers may refer to Bubnová et al. (1995), Radnóti (1995), Horányi et al. (1996) and Geleyn (1998) for a general overview. Special configuration of this model was prepared in order to fulfill the requirements of the MFSTEP (Mediterranean Forecasting System Towards Environmental Prediction) project.

ALADIN/MFSTEP runs at the horizontal resolution of $9 \mathrm{~km}$ and it has 37 levels. It is coupled with the ARPEGE global model. It is run in the pseudo-assimilation mode based on the long cut-off cycle of ARPEGE. According to the MFSTEP specifications, a production forecast is provided once a week up to the range of five days with hourly outputs. To provide a continuous forcing, hourly outputs from the assimilation cycle are available as well.

Since the aim was to provide high quality meso-scale atmospheric forcing data over the Mediterranean basin, we fo-

Correspondence to: R. Brožková

(radmila.brozkova@chmi.cz) cused on the forecasting problems linked to the presence of complex orography in the vicinity of the sea coast. At the same time we worked on the modifications and tuning of the physical parameterisation package of the model in order to improve forecast of the surface fluxes required for the forcing of basin and shelf models. When it was available we used the oceanographic data to validate and or retune the ALADIN/MFSTEP application.

In this way we achieved a combination of the already existing and new improvements, explained in the following sections and touching all main three parts of the ALADIN model: preparation of the initial conditions, dynamical core and physics.

\section{Blending the initial conditions}

ALADIN/MFSTEP receives its lateral boundary conditions from the global model ARPEGE. Given the fact that ARPEGE uses the 4-DVar data assimilation method, Courtier et al. (1994), and that the domain of ALADIN/MFSTEP is not large enough to provide a sample for longer waves to be properly analysed, Berre (2000), a careful design of the limited area analysis is needed.

As a natural demand, ALADIN initial conditions should be properly coupled with the longer waves analysed by ARPEGE and if possible, additional information should be present in the small scale part of the spectra resolved thanks to the finer mesh of the limited area model. A simple technique, called blending, was designed to fulfil this task (Brožková et al., 2001). We know from the optimal estimation theory used in data assimilation that the background field, provided by the previous short integration of the model, is one important source of information to obtain an optimal estimate of the atmospheric state. This hypothesis is used in blending, where we blend the long wave part of the spectra from the 4-DVar analysis of the driving model with the

Published by Copernicus GmbH on behalf of the European Geosciences Union. 


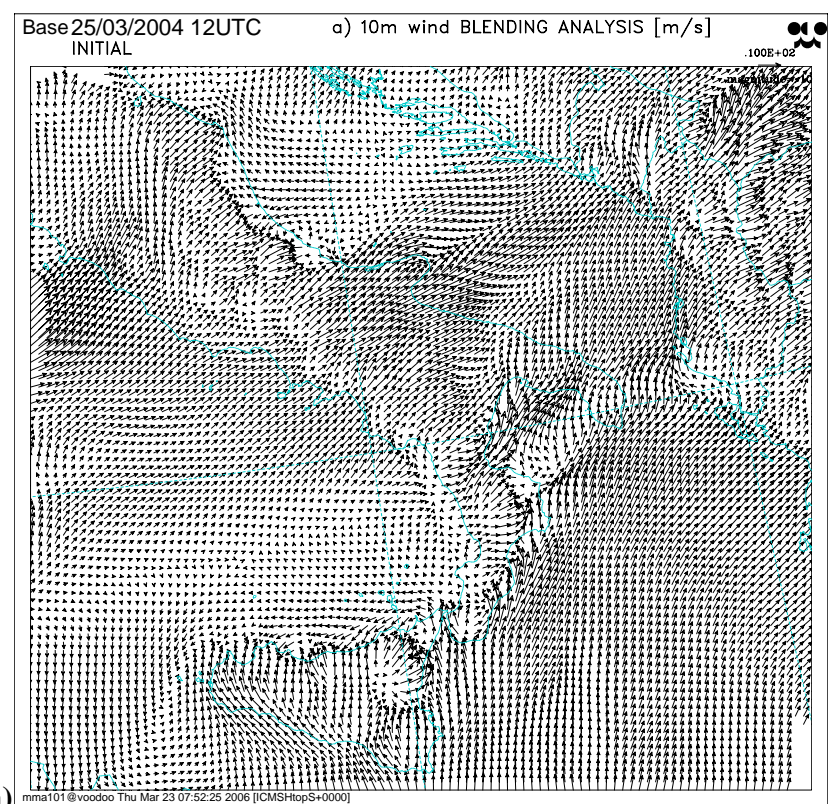

(a)

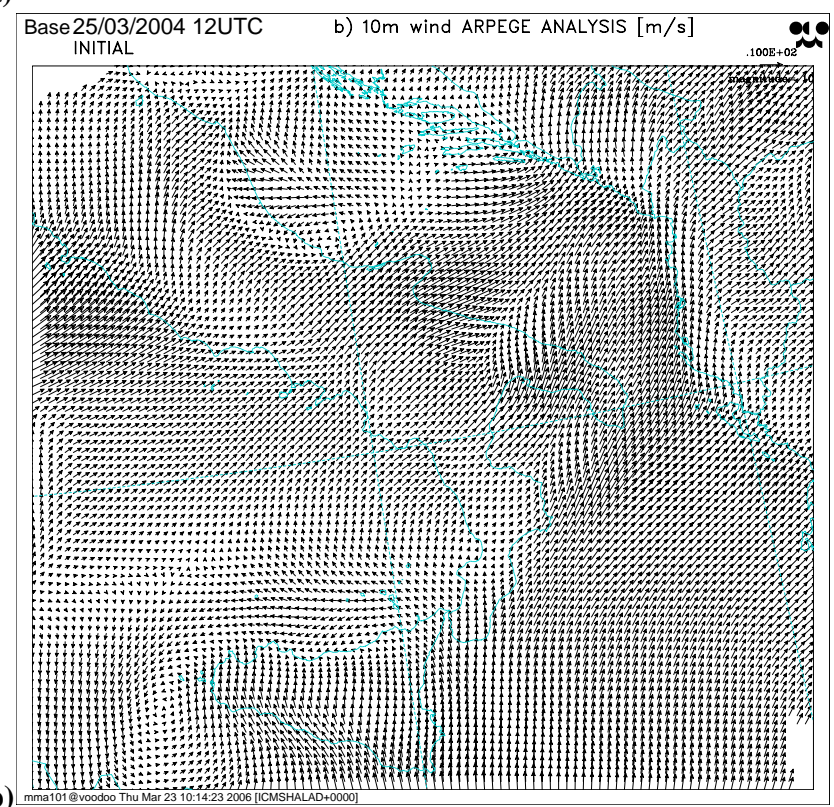

Fig. 1. Analysis of the wind field at $10 \mathrm{~m}$ above the ground, extracted from the MFSTEP domain and valid at 12:00 UTC on 25 March 2004: (a) ALADIN analysis using the blending method; (b) ARPEGE global model analysis on the ALADIN grid.

background field (or better to say guess) of the fine-scale limited area model.

Blending may also be seen as a special application of the incremental technique used in the 4-DVar data assimilation scheme (Courtier et al., 1994). Here also the analysis is not made for all resolved waves; the short part of the spectra is initialised from the background field. The reason is twofold: firstly the analysis algorithm is cheaper and converges faster; secondly the shortest waves are not easily analysed for various reasons and thus the background field is the most reliable source of information. The short waves also adapt reasonably quickly to the long wave part of the spectra which is a security in case the guess would have larger error than usual. Of course, the split between the analysed long wave part of the spectra and the short one must be properly tuned. This is true also for blending, where the ALADIN guess provides better information on the fine scales than the interpolated ARPEGE analysis which does not resolve these scales.

For the upper-air fields the blending is applied on the model spectra (both ARPEGE and ALADIN are spectral models) with the cut-off truncation separating the large scales analysed by ARPEGE and fine scales resolved in the guess of ALADIN. The blending method itself is implicit, using a digital filter scheme as a temporal and hence also spatial filter. The description of digital filter techniques for NWP may be found in Lynch and Huang (1992).

The symbolic equation of the spectral digital filter blending is

$A_{\text {ala }}=G_{\text {ala }}+\left\langle\left(A_{\text {arp }}^{\text {filter }}\right)_{T c}-\left(G_{\text {ala }}^{\text {filter }}\right)_{T c}\right\rangle_{\text {HighRes }}$

Where $A$ denotes the analysis, $G$ denotes the guess and $T c$ stands for the cut-off truncation. The filtering is made at the low spectral resolution $T c$ in order to remove small-scale noise or in other words to obtain a clean long wave state. The final sum of the fields is made at the high resolution of ALADIN/MFSTEP and the resulting analysis $A_{\text {ala }}$ is then initialised using a weak incremental digital filter. This means that only analysis increments are filtered as it follows

$A_{\text {ala }}^{b}=G_{\text {ala }}+I^{\text {filter }}=G_{\text {ala }}+\left(A_{\text {ala }}^{\text {filter }}-G_{\text {ala }}^{\text {filter }}\right)$

The upper script $b$ denotes the final balanced analysis; $I$ stands for the analysis increment.

Some soil prognostic variables are blended too, for which global model analysis increments are added to the fine scale guess with a weak relaxation towards the global model analysis, while some other fields are reinitialised directly from the guess. The sea surface temperature is initialised from the global model analysis.

The improvement in the initial state by blending is shown on Fig. 1. Fine scale sea and mountain breeze is well developed along the coast in case of the blended initial state compared to the initial state obtained from the coarser mesh global model. We see how blending significantly helps to reduce the spin-up of the meso-scale circulations in the first hours of integration. In addition, the atmospheric forcing provided from the computation of the guess within the blending cycle (the blended analysis is made every six hours) is rather well suited for more continuous warming up of sea models. 


\section{The nonlinear horizontal diffusion}

An original method using the properties of semi-Lagrangian interpolation operators in order to formulate a nonlinear horizontal diffusion was proposed by Váňa et al. (2001). It is a scheme suitable for spectral semi-implicit semi-Lagrangian models (so-called SISL class of models), where it would otherwise be technically difficult and expensive to implement locally varying diffusion coefficients.

The idea is here that the degree of interpolation operators, used for the semi-Lagrangian advection scheme, determines their damping properties. For example a linear interpolator is more diffusive than a rather accurate cubic interpolator. It is therefore possible to propose a diffusion scheme, combining the accurate and diffusive interpolators, which is then implemented within the basic semi-Lagrangian advection computations. Compared to other kinds of local and non-linear diffusion operators it is rather cheap. It is also fully stable thanks to its numerical algorithm.

The diffusion strength is controlled by a coefficient depending on the deformation of the wind field (the higher the deformation the bigger weight given to the more diffusive part of the operator). Of course, alike for any classical horizontal diffusion scheme, the tuning of this dependency takes into account the model's mesh-size, plus the latter's influence on the diagnostic of the deformation (Váňa, personal communication). In this way, the energy and enstrophy cascading properties are made as scale-independent as possible. The scheme then acts less and less, for a given atmospheric feature, when higher resolution allows to describe it within the scope of the well resolved model scales. The implementation as made in ALADIN/MFSTEP uses the horizontal wind field for the diagnostic of flow deformation, while the interpolators are 3-D. The method could be extended fully to the 3-D space if an appropriate 3-D flow deformation tensor would be computed. The importance of the local and non linear diffusion scheme is quite well seen already at the resolution of ALADIN/MFSTEP, compared to more simple linear diffusion scheme frequently used in spectral models. Thanks to its local and 3-D character SLHD (semi-Lagrangian horizontal diffusion) prevents from too intensive air mixing along the orographic slopes leading to spurious erosion of inversion layers. It is therefore physically more realistic. The local control by the flow deformation also ensures that the model does not overestimate a meso-scale cyclogenesis over the sea surface.

An example of such cyclogenesis over Black Sea is shown on Fig. 2. ALADIN/MFSTEP was able to simulate the development of a small scale cyclone comparing rather well with the satellite observation, but its intensity was too strong in the control experiment using the linear spectral diffusion. The experiment with the SLHD scheme still keeps the small scale cyclone in the forecast while its intensity is not any more pathological. This is confirmed by quite realistic structures of the potential vorticity anomaly near the surface and
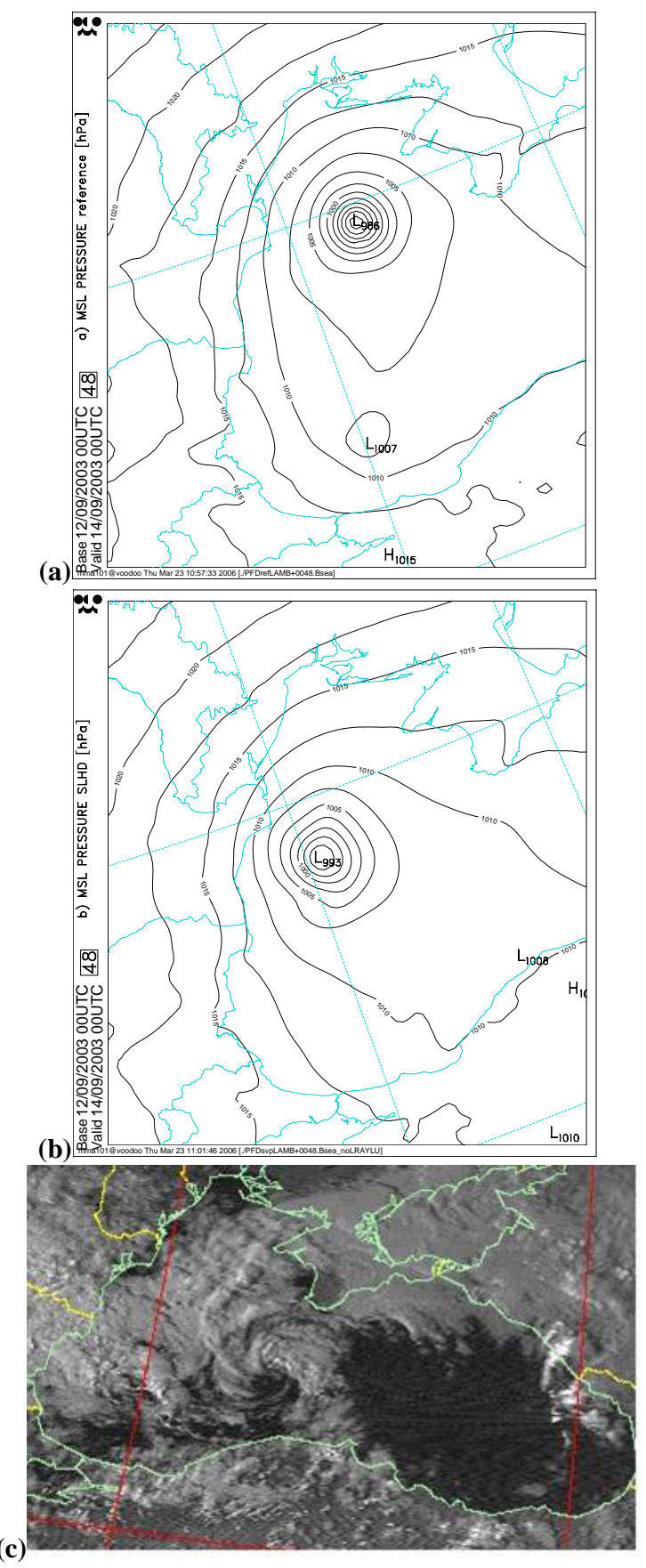

Fig. 2. ALADIN $48 \mathrm{~h}$ forecast of mean-sea-level pressure, valid at 00:00 UTC on 14 September 2003, zoomed over the western part of the Black Sea: (a) reference experiment; (b) experiment using the SLHD horizontal diffusion. Panel (c): NOAA satellite visible channel picture, valid at 14:27 UTC on 13 September 2003, showing the presence of a developing meso-scale cyclone.

the tropopause (not shown). One may argue that a quasiperfect physical forcing within the diabatic part of the model would make this curative role of SLHD unnecessary. It is 


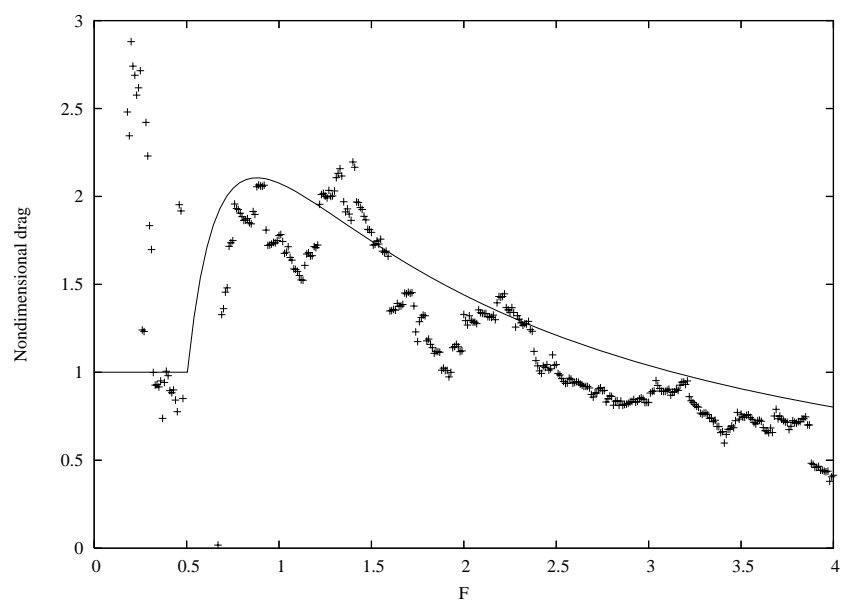

Fig. 3. The non-dimensional drag as a function of the inverse Froude number $F$. Solid line: theoretical dependency; crosses: diagnostic result from the pseudo-academic experiment over the Alps at the horizontal resolution of $10 \mathrm{~km}$.

however a general feature of parameterisation packages that those exciting less the physics-dynamics interplay are also those most frequently missing actual extreme events. In that sense, SLHD can be seen both as a more realistic flow dependent damping operator and as a control mechanism for improving the hit vs. false alarm rate concerning intense events.

\section{Improvements in physics}

As it was already stated in the introduction, we focused on the developments leading to a better description of flow in mountainous and coastal areas as well as to improvement of radiation fluxes, cloudiness and flow over the sea surface. The content of these developments is explained below.

\subsection{Mountain drag and lift scheme}

The so-called envelope orography proposed by Wallace et al. (1983) was frequently used in the models to cope with modelling of sub-grid scale orographic effects and this was the case for the ALADIN model till recently. The parameterisation schemes describing the forcing of unresolved mountains permit today to get-rid of the artificial enhancement of peaks of the envelope orography. Therefore when preparing the ALADIN/MFSTEP application we chose to abandon the envelope orography concept and for that we needed to improve, tune and validate a new parameterisation scheme of mountain drag and lift.

The original scheme treated the linear part of the drag, acting at higher atmospheric levels, according to Boer et al. (1984) and the form drag, acting at low levels, according to Lott and Miller (1997). When removing the envelope from the orography we needed to seek a better tuning of the surface drag and to add the so-called lift effect, following Lott (1999) but with some revisions. We shall mention here only the most important ingredients of the new version of the scheme.

The first is the computation of the surface drag in dependency on the inverse critical Froude number $F c$, a non dimensional mountain height, depending on the effective mountain height, static stability and wind speed. If the actual inverse Froude number $F$ is lower than a critical value (a tuning parameter of the scheme), the lower part of the flow does not have to go around the obstacle and the surface stress is equal to the wave part of the stress and it is computed according to the linear theory, Bougeault (2001). In the opposite case there is in addition the form drag at work and it is expressed according to Scinocca and McFarlane (2000). In our scheme we have chosen to use a geometrical average between the depth of the perturbed flow generating the gravity waves and the effective mountain height for computing the wave part of the stress. Like that the expression for the ratio of the total stress and its wave part becomes nicely compact and linear with respect to the effective mountain height. Compared to the old scheme, the total surface drag is increased roughly by the factor $F / F c$ when the form drag is acting and under the opposite conditions it is diminished following the same factor. Figure 3 shows quite a nice agreement of the new nondimensional stress with the theoretical one in dependency on the inverse Froude number as it was diagnosed in the model over the Alps within the preliminary tests of the scheme.

The second change consists in a new vertical partition of the wave drag deposition. Adapting Lindzen (1981) proposal to the above choices for the surface drag value, only the purely wave part of the total surface stress is deposited above the first critical level of the flow, while the remaining stress part is deposited linearly between the surface and this critical level.

The third ingredient is the introduction of the lift effect in order to compensate for a missing volume of the resolved orography (which was before compensated by the envelope orography). Here we corrected the original implementation by making the lift force to act in a correct direction. In the previous scheme this force was orthogonal to the actual wind while it should be orthogonal to the geostrophic wind, for which we now compute a first order evaluation under a hypothesis of stationarity.

The new scheme was first validated and tuned using the pseudo-academic 3-D experiments with real orography at various horizontal resolutions. The first operational implementation was made in ALADIN/MFSTEP, where the quality of the forecast was checked both for the old scheme with the envelope orography and for the new scheme without the use of the envelope. We obtained about the same quality of the general forecast. Precipitation amounts at the orographic slopes are now better predicted since the peaks are not any more artificially enhanced. On the other hand we have got a weak negative bias of the wind speed at the surface. This 
is fortunately compensated by a better structure of the flow around the mountains.

\subsection{Radiation scheme}

The radiation scheme used in ALADIN is adapted from Ritter and Geleyn (1992). In the ALADIN/MFSTEP we have introduced several improvements to this scheme in its thermal part formulated on the basis of the net exchange rate formalism (Green, 1967).

The main novelty is the following. When knowing the primary thermal exchange terms (cooling to space, exchange with surface and exchange with the adjacent levels), we have already the minimum and maximum optical thicknesses under which a given air layer is seen from any part of the atmosphere (when only gaseous effects are considered). Therefore with only a minor additional computation it is possible to bracket the true optical thickness for the remaining thermal exchange terms.

The practical implementation then requires finding out the interpolation coefficients retrieving the best estimate from the two bracketing values. At first place we verified that results are more accurate when the interpolation is applied to the fluxes rather than to the cooling rates. Then the next step was to parameterise the local values of the interpolation weight, using a statistical approach. After stratifying a large dataset, a parameterisation of the weight was proposed (Geleyn et al., 2005), where the statistical fit turns out to be rather simple, having only two tuning constants. The practical tests in ALADIN/MFSTEP have shown a nice improvement of the scores with respect to the simpler old scheme which so to say used only the lower limit of the bracket.

Four other smaller changes were introduced to the scheme. There are now "exact" corrective terms of the thermal exchange fluxes between the adjacent atmospheric levels as if there was no scattering. These terms are of course not accounted in the search of the interpolation coefficients of the bracketing scheme explained above. Computation of this correction takes into account the local temperature gradient instead of assuming that each model layer is isothermal. In addition, the non-linearity of the gaseous transmission function is used for the adjacent levels exchange terms. This means to add one more computation of the gaseous transmission per layer but the scheme remains still very efficient. Finally, the split-implicit mode treatment of the time-step to linearly estimate the induced changes of the black-body radiation term $\sigma T^{4}$ for all the four principal exchange terms ensures the numerical stability of the bracketing scheme.

\subsection{Low level cloudiness}

Cloudiness in the model depends on the diagnosed liquid or ice water content. The functional dependency follows the proposal of Xu and Randall (1996). For ALADIN/MFSTEP we retuned the part of the scheme concerning the stratiform clouds.

It turned out to be necessary because the stratiform clouds were often either saturated or not formed at all, with very small amounts of intermediate clouds. While this "binary" behaviour did not matter much for the general model scores, it had a direct consequence on the intensity of radiation fluxes. In presence of clouds the surface solar flux was too weak as it turned out within the MFSTEP scientific validation period of January 2003. In the scheme an instantaneous super-saturation of the air layer of a certain thickness is estimated from the local saturation multiplied by the so called critical relative humidity vertical profile. A new shape and tuning of the critical relative humidity profile $H u_{c}$ was proposed to match better the cloud observations and it reads

$$
\begin{aligned}
H u_{c}(\eta)= & 1-1.4 \eta(1-\eta) /((1-0.6(\eta-0.5)) \\
& (1+1.1(\eta-0.5)))
\end{aligned}
$$

Here $\eta$ is the vertical coordinate of the model, varying from 1 (surface) to 0 (top of the atmosphere). This new tuning, applied together with the maximum-random overlap hypothesis for the cloud geometry, lead to a significant improvement of the predicted cloud amounts. The binary-like behaviour of the clouds formation was removed and consequently we obtained more realistic radiation fluxes. However one weakness was not yet cured. The model was not capturing very well the low level inversion cloudiness, including marine stratus, and thus the $2 \mathrm{~m}$ temperature forecast had too strong diurnal cycle in the typical inversion conditions.

A special treatment for this type of clouds was therefore added to the scheme. The idea is rather simple: when a temperature inversion layer is detected, the temperature used for computing the saturation function is cooled a bit in order to help the cloud formation process. This method has of course a positive feedback, since the presence of clouds reinforces the inversion.

With respect to the implementation algorithm, there are two tuning constants. The first one is the minimum thickness of the inversion layer required for the activation of this scheme. This is to avoid a flip-flap effect when going from one vertical layer to the next and the constant was tuned to the geopotential thickness of $1750 \mathrm{~J} / \mathrm{kg}$. The temperature shift itself is then proportional to the diagnosed temperature gradient as

$\phi_{0} \partial T / \partial \phi$

with the coefficient $\phi_{0}$ tuned to $1250 \mathrm{~J} / \mathrm{kg}$. The effect of the stratus treatment can be seen on Fig. 4 showing a typical anticyclonic situation with presence of low level clouds. On the polar orbit satellite picture these clouds have a typical yellowish colour, while higher level clouds are rather white. The reference experiment forecast clearly misses most of the stratus clouds while the test experiment with the new stratus treatment included provides more realistic, although not yet perfect, low-level cloudiness forecast. 


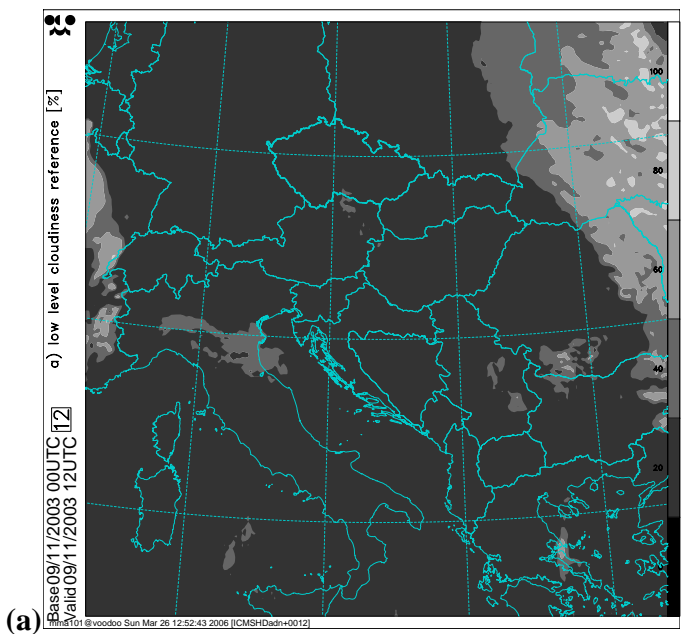

(a)

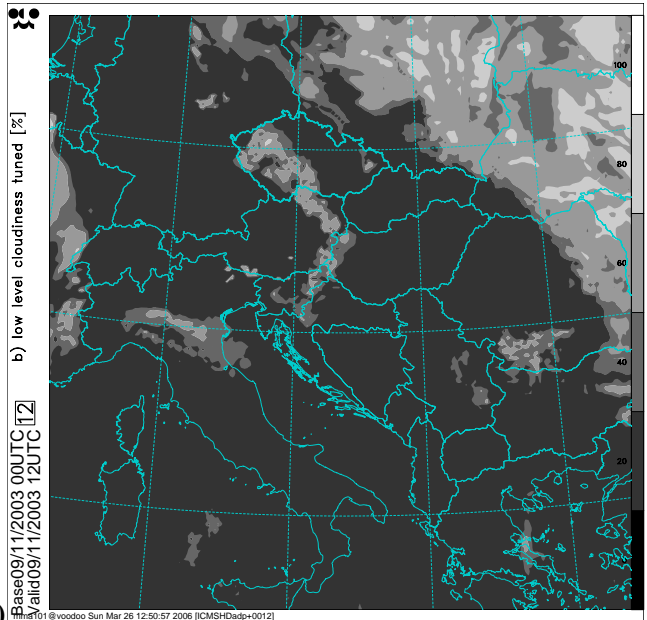

(b)

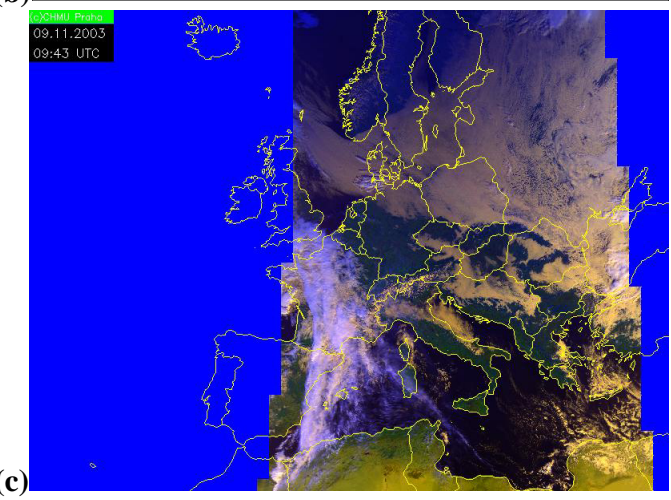

Fig. 4. ALADIN $12 \mathrm{~h}$ forecast of low level cloudiness, valid at 12:00 UTC on 9 November 2003: (a) reference experiment; (b) experiment with the updated cloudiness scheme. Panel (c): NOAA visible channel satellite picture, valid at 09:34 UTC on 9 November 2003.

\subsection{Moist gustiness}

According to bulk aerodynamic transfer theory, the vertical turbulent fluxes are locally proportional to the wind shear.
For surface fluxes it means that they are proportional to the wind speed at the lowest model level. However, when using the averaged wind speed representing the model gridbox (this quantity is known by the model) the resulting effect would be underestimated. This is because the magnitude of the averaged wind vector can be significantly smaller than the average magnitude of sub-grid vectors. Such conditions are fulfilled mainly when the mean wind is close to zero and especially in the tropics where the weak Coriolis force allows for randomly generated gusts.

From the triangle inequality it can be derived that the scalar average is bigger or equal to the vectorial one:

$\frac{1}{N} \sum_{i=1}^{N}\left|\boldsymbol{v}_{i}\right| \geq \frac{1}{N}\left|\sum_{i=1}^{N} \boldsymbol{v}_{i}\right|$

Therefore the difference between the computed and theoretically expected wind speed, which represents the effect of sub-grid horizontal wind variability, so-called gustiness, should be parameterized in the model. In case of dry convective origin the gustiness effect is already treated in the planetary boundary layer parameterization by Louis (1979) through an additional term in the Charnock formula

$z_{0}=\frac{C_{k}}{g} u_{*}^{2}+\frac{C_{d}}{C_{d n}} z_{0}^{m}$

In the second term $C_{d}$ is the drag coefficient, $C_{d n}$ is its neutral value and $z_{0}^{m}$ is a tuneable critical value for the momentum roughness length. We see that this additional term is proportional to the stability modulation factor, in order to account for gusty conditions.

However, the case of moist convection as the origin of gusts was not treated yet in the model and this missing process was partly compensated by some overtuning of its dry gustiness counterpart.

The parameterization of additional moist gustiness follows the proposal by Redelsperger et al. (2000) based on TOGA-COARE (Tropical Ocean Global Atmosphere - Coupled Ocean Atmosphere Response Experiment) measurements. According to the original idea, the moist gustiness would be accounted only over the sea surface by augmenting the drag coefficient. This is done by a Pythagorean addition to the modulus of the low level wind in dependency on the precipitation flux $P_{r}$

$$
|\boldsymbol{U}|=\sqrt{|\boldsymbol{U}|^{2}+U_{g}^{2}}
$$

Where we have

$$
U_{g}=\ln \left(1+6.69 P_{r}-0.476 P_{r}^{2}\right)
$$

The implementation of this idea in ALADIN required some additional adjustments, since the logarithmic formula used in Eq. (7) did not represent the whole scale of materialized precipitation fluxes. A new formula for the wind offset was chosen, in order to fulfil the following properties: an asymptotic 
behaviour for higher precipitation fluxes, independence on the height of the lowest model level, a possibility to extend this parameterisation also to land and upper levels. These conditions are met by adding to the modulus of the stress divided by density the square of a precipitation dependent wind offset, which amounts to multiply the drag and/or exchange coefficients by the following quantity

$\sqrt{1+\left(\tilde{U}\left(\frac{P_{r}}{P_{r}+P_{r}^{0}}\right)^{\gamma}\right)^{2} \frac{\rho}{\left|\boldsymbol{F}_{\boldsymbol{u}}\right|}}$

$\boldsymbol{F}_{\boldsymbol{u}}$ stands for wind stress vector.

There are three tuning constants:

$\tilde{U}$, which should be of the order of the typical surface friction velocity;

$\gamma$, which has to be chosen following the TOGA-COARE results;

$P_{r}^{0}$, which is a typical steadily strong precipitation flux.

The tuning is respectively $0.125 \mathrm{~m} / \mathrm{s}, 0.8$ and $1.15 \mathrm{E}-$ $04 \mathrm{~kg} / \mathrm{m}^{2} \mathrm{~s}$ (this is about $10 \mathrm{~mm} /$ day).

\subsection{Sea roughness length tuning}

Given the importance of the surface sensible and latent heat fluxes for the oceanic forcing we decided to tune the computation of the roughness length values over sea. We decided to take two references. The MFSTEP validation data set of Ramos Buarque et al. (2004) and the ECMWF (European Centre for Medium-range Weather Forecasts) operational fluxes, all available for the January 2003 specific MFSTEP scientific validation period. After a preliminary examination of all relevant fluxes for the whole month, we elected to concentrate on the latent heat flux and on the first five days of the month (with a set of situations representative of the various conditions of the whole period).

It must now be mentioned that, already at the beginning of the MFSTEP work, an important effect was included which did not exist up to then in the ALADIN model: the differentiation over sea of the mixing lengths for momentum on the one hand (computed according to Eq. 5) and for heat and moisture on the other hand. This is meant to simulate the observed fact that, when the wind increases, the sensible and latent heat fluxes correspond to a surface exchange coefficient $C_{h}$ which value saturates to an asymptote while the corresponding coefficient for momentum, $C_{d}$, continues to increase, via the effect of the Charnock formula. The chosen expression to obtain this effect is

$z_{0}^{h}=z_{0}^{m} \exp \left(-u_{*} / u_{*}^{0}\right)$

With this formula and the modified Charnock one, the latent heat fluxes depend on some overtuning of the critical value for the momentum roughness length (1.5 E-04 being the starting value) and of the reference friction velocity value for the above "differentiation" equation (we started with an empirically evaluated value of 0.25 ).
LATENT HEAT (1.1. - 5.1. 2003)

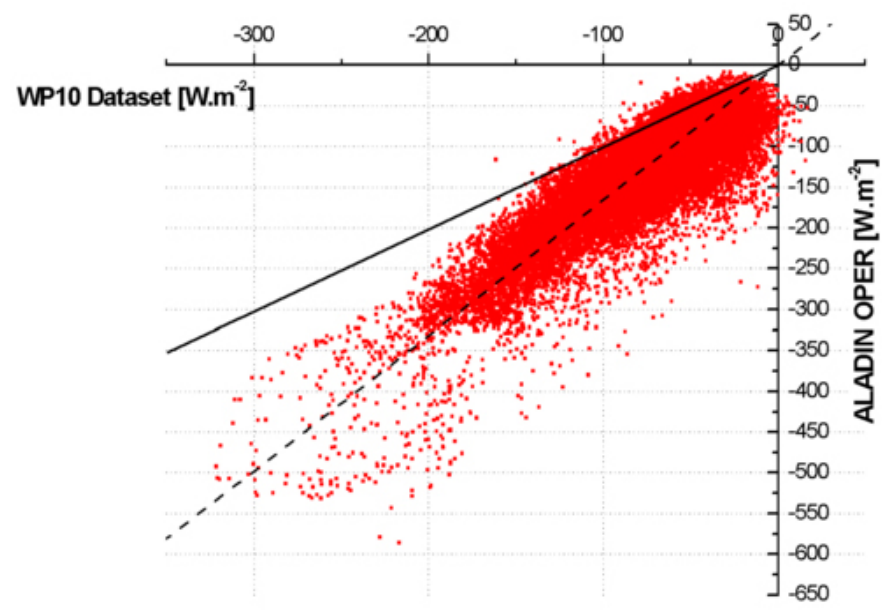

Fig. 5. Dispersion diagram for the latent heat flux: ALADIN configuration prior to the sea roughness tuning is compared to the MFSTEP-WP10 dataset.

The dispersion diagram (Fig. 5) obtained for the comparison of the non-tuned situation with the so-called "WP10" MFSTEP data set (for the latent heat fluxes) indicates two weaknesses: a non-zero crossing of the zero line (with about $50 \mathrm{~W} / \mathrm{m}^{2}$ difference) and an increase of this bias with increasing values. The latter is especially true for the highest flux values, where even the least-square fit of the whole cloud of points ceases to be representative of the increased bias (too strong fluxes by higher winds and thus a likely still too high value of the reference friction velocity - the previous ALADIN situation is equivalent to an infinite value for this parameter).

The comparison of the same set of ALADIN/MFSTEP results with the ECMWF modelling reference (Fig. 6) helps to put these results in perspective: The general trend of the least-square fit curve indicates a quasi constant bias but with still a shift, albeit smaller, at the zero intercept, this indicating a probably too high dry gustiness term in ALADIN, and the same deviations at high flux values.

Following these findings a tuning of 0.1 for the reference friction velocity and of $1.0 \mathrm{E}-04$ for the critical value of the momentum roughness length were found as an acceptable compromise to mitigate both problems with respect to the references.

Indeed the new comparison with the ECMWF data (Fig. 7) gives now a $0 . / 0$. welcome behaviour for the weak fluxes and a more consistent behaviour for the whole range of values of the cloud of points, including for the highest values, even if this is obtained at the expense of a far less "parallel" behaviour of the two data sets. On the contrary (Fig. 8), the parallelism is now nicely there with respect to the WP10 data set, the shift at the zero line intercept being down to $30 \mathrm{~W} / \mathrm{m}^{2}$ 
LATENT HEAT (1.1. - 5.1. 2003)

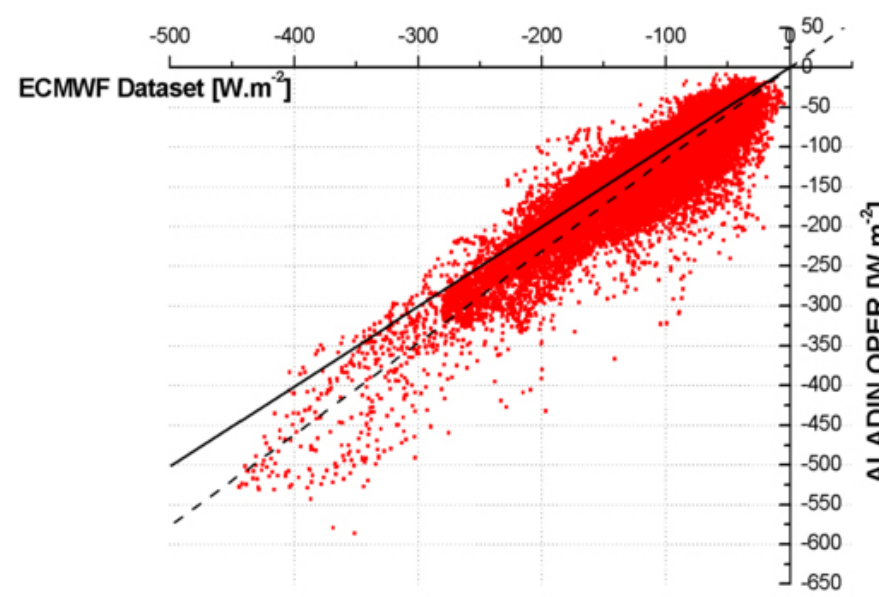

Fig. 6. Dispersion diagram for the latent heat flux: ALADIN configuration prior to the sea roughness tuning is compared to the ECMWF dataset.

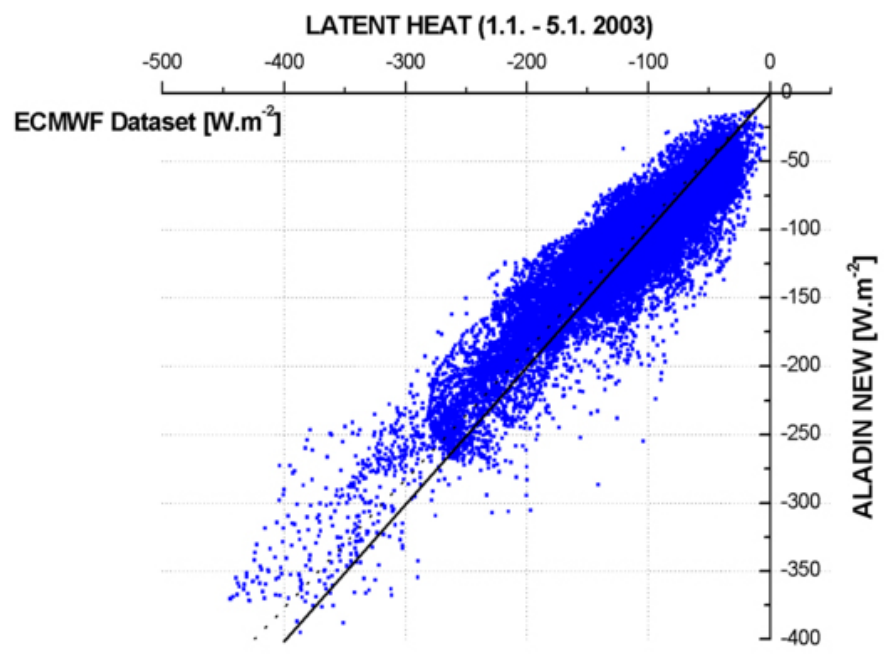

Fig. 7. Dispersion diagram for the latent heat flux: the tuned ALADIN configuration in comparison with the ECMWF dataset.

and this "shift" being kept constant all along the range of flux intensities.

One may here argue that this shift, common with the ECMWF results at low wind speeds, is due to the use of purely local-type computations for the creation of the MFSTEP validation data-set, something that indeed neglects the gustiness-type flux enhancement within a grid-box, linked to the intrinsic sub-grid variance of the wind strength. Admitting this explanation, our new tuning appears very satisfying: a good statistical relation with the WP10 data set plus a constant empirical gustiness correction of $30 \mathrm{~W} / \mathrm{m}^{2}$ and an homogeneous even if linearly biased comparison with the less relevant (in terms of absolute values) ECWMF figures.

Moist gustiness parameterisation together with sea rough-
LATENT HEAT (1.1. - 5.1. 2003)

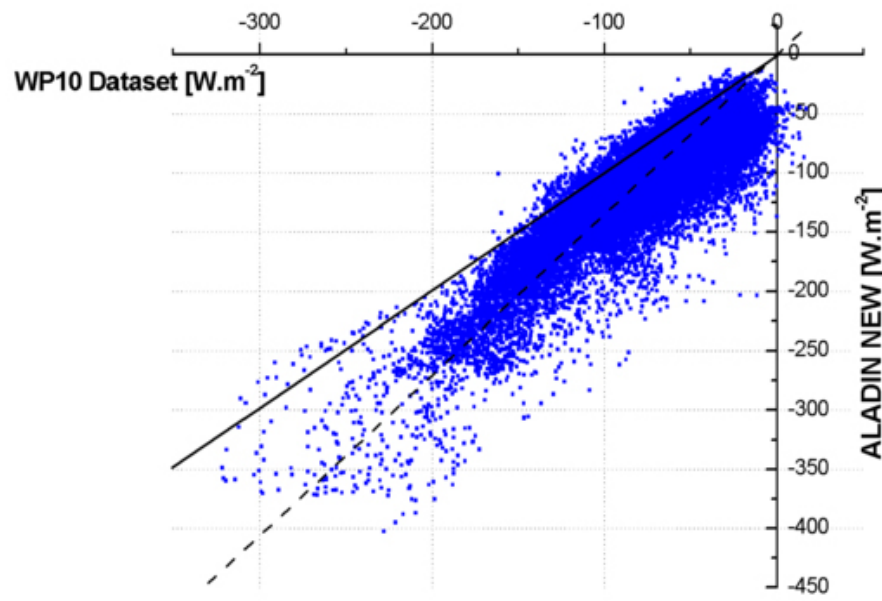

Fig. 8. Dispersion diagram for the latent heat flux: the tuned ALADIN configuration in comparison with the MFSTEP-WP10 dataset.

ness length tuning help to reach more realistic simulation of the cyclogenesis over the sea. When tested on the Black Sea cyclogenesis case it had roughly the same amount of beneficial impact as the SLHD diffusion shown on Fig. 2 compared to the control experiment. But of course the combination of these ingredients does not mean to sum up their individual effects. Given the construction of the SLHD diffusion, which strength is given by the wind field deformation, it acts much less in presence of improved parameterisation of turbulence over the sea.

\section{Conclusions}

The preparation of ALADIN/MFSTEP fostered a specific effort resulting in a set of changes applied in its operational realisation. Although each of these ingredients alone brought a small improvement, the ensemble represents a non negligible improvement of the basic model. The main outcomes can be synthesised as follows. The blending approach for coupling a global data assimilation cycle at large-scale without reanalysing at the limited area small-scale found a favourable ground in the computation of near costal atmosphere-ocean fluxes. A more scale-selective new concept for the lateral diffusion helped having a better control over the intensity of rapidly deepening Mediterranean cyclones. Radiative and sub-grid momentum fluxes were modified in order to improve their MFSTEP usefulness. The diagnostic computation of cloudiness was retuned and enhanced in order to meet some MFSTEP oceanographic users' requirements. Last but not least, more sophisticated formulations for the surface turbulent exchanges were developed and tuned with respect to MFSTEP specific data. 
Acknowledgements. The authors are thankful to all the MFSTEP colleagues involved in the validation of atmospheric forcing and to the ALADIN community. The work was enabled thanks to the MFSTEP project, European contract EVK3-CT-2002-00075.

Edited by: N. Pinardi

\section{References}

Berre, L.: Estimation of synoptic and mesoscale forecast error covariances in a limited area model, Mon. Wea. Rev., 128, 644667, 2000.

Boer, G. J., Mc Farlane, N. A., Laprise, R., Henderson, J. D., and Blanchet, J.-P.: The Canadian Climate Centre spectral atmospheric general circulation model, Atmos.-Ocean, 22, 397-429, 1984.

Bougeault, P.: Sub-grid scale orography parameterisations. ECMWF Seminar Proceedings on "Key issues in the parameterisation of subgrid physical processes", 3-7 September 2001, 53-69, 2001.

Brožková, R., Klaric, D., Ivatek-Sahdan, S., Geleyn, J.-F., Cassé, V., Široká, M., Radnóti, G., Janoušek, M., Stadlbacher, K., and Seidl, H.: DFI blending, an alternative tool for preparation of the initial conditions for LAM, PWRP Report Series No. 31, WMOTD No. 1064, 1.7., 2001.

Bubnová, R., Hello, G., Bénard, P., and Geleyn, J.-F.: Integration of the fully elastic equations cast in the hydrostatic pressure terrainfollowing coordinate in the framework of the ALADIN NWP system, Mon. Wea. Rev., 123, 515-535, 1995.

Courtier, P., Thépaut, J.-N., and Hollingsworth, A.: A strategy for operational implementation of 4D-Var using an incremental approach, Q. J. R. Meteorol. Soc., 120, 1367-1387, 1994.

Geleyn, J.-F.: Adaptation of spectral methods to non-uniform mapping (global and local), ECMWF Seminar Proceedings on "Recent developments in numerical methods for atmospheric modeling", 7-11 September 1998, 226-265, 1998.

Geleyn, J.-F., Fournier, R., Hello, G., and Pristov, N.: A new "bracketing" technique for a flexible and economical computation of thermal radiative fluxes, on the basis of the Net Exchange Rate (NER) formalism, WGNE Blue Book, 2005 Edition, 4-07, 2005 .
Green, J. S. A.: Division of radiative streams into internal transfer and cooling to space, Q. J. R. Meteorol. Soc., 93, 371-372, 1967.

Horányi, A., Ihász, I., and Radnóti, G.: ARPEGE/ALADIN: A numerical weather prediction model for Central Europe with the participation of the Hungarian Meteorological Service, Idöjárás, 100, 277-301, 1996.

Lindzen, R. S.: Turbulence and stress owing to gravity wave and tidal breakdown, J. Geophys. Res., 86, 9707-9714, 1981.

Lott, F. and Miller, M. J.: A new sub-grid scale orographic drag parameterization: Its formulation and testing, Q. J. R. Meteorol. Soc., 123, 101-127, 1997.

Lott, F.: Alleviation of stationary biases in a GCM through a mountain drag parameterization scheme and a simple representation of mountain lift forces, Mon. Wea. Rev., 127, 788-801, 1999.

Louis, J.-F.: A parametric model of vertical eddy fluxes in the atmosphere, Boundary-layer Meteorol., 17, 187-202, 1979.

Lynch, P. and Huang, X.-Y.: Initialization of the HIRLAM model using a digital filter, Mon. Wea. Rev., 120, 1019-1034, 1992.

Radnóti, G.: Comments on "A spectral limited-area formulation with time-dependent boundary conditions applied to the shallowwater equations". Mon. Wea. Rev., 123, 3122-3123, 1995.

Ramos Buarque, S. and Caniaux, G.: High-resolution gridded airsea surface fluxes over the Mediterranean basin by a synthesis approach, Météo-France's contribution report to MFSTEP-WP10, subtask 10320, 20 p., 2004.

Redelsperger, J.-L., Guichard, F., and Mandon, S.: A parameterization of mesoscale enhancement of surface fluxes for large scale models, J. Climate, 13, 402-421, 2000.

Ritter, B. and Geleyn, J.-F.: A comprehensive radiation scheme for numerical weather prediction models with potential applications in climate simulations, Mon. Wea. Rev., 120, 303-325, 1992.

Scinocca, J. F. and Mc Farlane, N. A.: The parameterization of drag induced by stratified flow over anisotropic orography, Q. J. R. Meteorol. Soc., 126, 2353-2393, 2000.

Váňa, F., Bénard, P., and Geleyn, J.-F.: Nonlinear horizontal diffusion formulation in spectral semi-Lagrangian models, PWPR Report Series No. 31, WMO-TD No. 1064, 5.44., 2001.

Wallace, J. M., Tibaldi, S., and Simmons, A. J.: Reduction of systematic forecast errors in the ECMWF model through the introduction of an envelope orography, Q. J. R. Meteorol. Soc., 109, 683-717, 1983.

Xu, K. M. and Randall, D.: A semi-empirical cloudiness parameterization for use in climate models, J. Atmos. Sci., 53, 3084-3102. 